Forschende

Komplementärmedizin una Klassische Naturheilkunde

\section{Tagungen und Kongresse $\cdot$ Meetings and Conferences}

Forsch Komplementärmed Klass Naturheilkd 2002;9:128-129
1...................

09.05.-12.05.2002

Freiburg i.Br.

Deutschland

\subsection{5.-02.06.2002}

Baden-Baden

Deutschland
International Congress of Applied Kinesiology - ICAK

\section{6. Ärztliche Weiterbildungswoche Baden-Baden}

Spirituelle und transpersonale Dimensionen der Psychotherapie

30.05.-02.06.2002

Bad Kissingen

Deutschland

\subsection{6.-08.06.2002 \\ Köln}

Deutschland

..................

08.06.-09.06.2002

Bad Brückenau

Deutschland

[..................

08.06.-09.06.2002

Berlin

Deutschland

[.................

06.07.-07.07.2002

Berlin

Deutschland

[.................

25.08.-31.08.2002

Pörtschach/

Wörthersee

Österreich

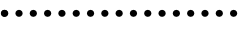

22.09.-29.09.2002

Überlingen

Deutschland

\section{Stoffwechsel-Tagung der ÄGHE}

\section{Immunologische Diagnostik und Therapie}

\section{Rechtliche und ethische Aspekte, Psychoonkologie}

51. Internationaler interdisziplinärer Seminarkongress für ärztliche Fortbildung

Summer School für Klassische Naturheilkunde
Auskunft: Comed $\mathrm{GmbH}$

Rolandstr. 63

D-50677 Köln

Tel. +49 221 801100-0, Fax -29

E-mail info@comed-kongresse.de

www.comed-kongresse.de

Auskunft: Sandra Jones

Karl F. Haug Verlag

Fritz-Frey-Str. 21

D-69121 Heidelberg

Tel. +49 6221 456450-7, Fax -0

E-mail medwoche@medizinverlag.de

www.medwoche.de

Auskunft: Albert Pietzko

Zentrum für Gesundheit

Hennebergstr. 22

D-97711 Massbach

Tel. +49 9735-695, Fax -367

Auskunft: Organisationsteam/AK Köln

HP Horst Büttner

Schwanenmarkt 8 a

D-40213 Düsseldorf

Tel. +49 211/328102, Fax +49 234/460113

E-mail HoBüttnerErk@t-online.deakmweil

\section{Auskunft:}

Malteser-Klinik Dr. von Weckbecker

Dres. med. Eva u. Norbert Lischka

Rupprechtstr. 20

D-97769 Bad Brückenau

E-mail mk_vonweckbecker.mtg@t-online.de

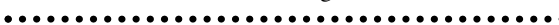

Auskunft: SynMed $®$ Institut und Verlag

Mommsenstr. 55

D-10629 Berlin

Tel. +49 30/785-7151, Fax -8212

E-mail kunosynmed@aol.com

www.akodh.de

www.synmed.netakmweil@akmcongress.com

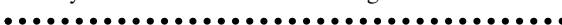

Auskunft: SynMed ${ }^{\circledR}$ Institut und Verlag

Mommsenstr. 55

D-10629 Berlin

Tel. +49 30/785-7151, Fax -8212

E-mail kunosynmed@aol.com

www.akodh.de

www.synmed.net

Auskunft: BDI e.V.

Kongressabteilung

Schöne Aussicht 5

D-65193 Wiesbaden

Tel. +49 611 18133-22, Fax -23

E-mail info@bdi.de

www.bdi.de

Auskunft: ESCNM

European Society for Classical Natural Medicine

Frau Dr. F. Wilhelmi de Toledo

Postfach 101165

D-88641 Überlingen

Tel. +49 7661 807-0, Fax -889

www.summer-school.de

\title{
KARGER
}

(C) 2002 S. Karger GmbH, Freiburg 
27.09.-29.09.2002

Überlingen

Deutschland

\subsection{9.-29.09.2002}

Berlin

Deutschland

\subsection{0.-09.10.2002}

Freudenstadt

Deutschland

06.10.-12.10.2002
Bad Pyrmont
Deutschland

\subsection{0.-13.10.2002}

Berlin

Deutschland

\subsection{0.-01.11.2002}

Baden-Baden

Deutschland

\section{..................}

30.10.-03.11.2002

Schönau

Deutschland

\section{...............}

09.11.-10.11.2002

Berlin

Deutschland

\section{................}

23.11.-24.11.2002

Berlin

Deutschland

\section{...............}

30.11.-01.12.2002

München

Deutschland

\section{Bodensee-Konferenz für klassische Naturheilkunde}

thoden

How to do, Anwendungsfragen immunbiologischer Medikamente und Methoden

Kongress des Zentralverbandes der Ärzte für Naturheilverfahren (ZÄN)

\section{Internationaler interdisziplinärer Seminarkongress für ärztliche} Fortbildung

\section{Onkologisches Notfallseminar}

\section{Medizinische Woche}

KURS A zur Erlangung der Zusatzbezeichnung Naturheilverfahren des Berufsverbandes Deutscher Internisten e.V.

\section{Hyperthermie}

.

\section{Klinis} nom (aktuelle Strategien in Diagnostik und Therapie, State of the Art in

\author{
Auskunft: ESCNM \\ Frau Dr. F. Wilhelmi de Toledo \\ Postfach 101165 \\ D-88641 Überlingen \\ Tel. +49 7661 807-0, Fax -889 \\ www.escnm.de
}

European Society for Classical Natural Medicine

Auskunft: SynMed ${ }^{\circledR}$ Institut und Verlag

Mommsenstr. 55

D-10629 Berlin

Tel. +49 30/785-7151, Fax -8212

E-mail kunosynmed@aol.com

www.akodh.de

www.synmed.netakmweil@akmcongress.com

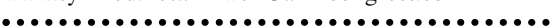

Auskunft: ZÄN

Am Promenadenplatz 1

D-72250 Freudenstadt

Tel. +49 7441 91858-0, Fax -22

E-mail ZAEN-Freudenstadt@t-online.de

www.zaen.org

Auskunft: BDI e.V

Kongressabteilung

Schöne Aussicht 5

D-65193 Wiesbaden

Tel. +49 611 18133-22, Fax -23

E-mail info@bdi.de

www.bdi.de

Auskunft: SynMed ${ }^{\circledR}$ Institut und Verlag

Mommsenstr. 55

D-10629 Berlin

Tel. +49 30/785-7151, Fax -8212

E-mail kunosynmed@aol.com

www.akodh.de

www.synmed.netakmweil@akmcongress.com

Auskunft: Ärzteges. f. Erfahrungsheilkunde e.V.

Fritz-Frey-Str. 21

D-69121 Heidelberg

Tel. +49 6221 489-469, Fax -202

Auskunft: BDI e.V.

Kongressabteilung

Schöne Aussicht 5

D-65193 Wiesbaden

Tel. +49 611 18133-22, Fax -23

E-mail info@bdi.de

www.bdi.de

Auskunft: SynMed ${ }^{\circledR}$ Institut und Verlag

Mommsenstr. 55

D-10629 Berlin

Tel. +49 30/785-7151, Fax -8212

E-mail kunosynmed@aol.com

www.akodh.de

www.synmed.net

Auskunft: SynMed ${ }^{\circledR}$ Institut und Verlag

Mommsenstr. 55

D-10629 Berlin

Tel. +49 30/785-7151, Fax -8212

E-mail kunosynmed@aol.com

www.akodh.de

www.synmed.net

\section{ZÄN-Adventskongress}

Auskunft: Z⿱艹̈N

Am Promenadenplatz 1

D-72250 Freudenstadt

Tel. + 497441 91858-0, Fax -22

E-mail ZAEN-Freudenstadt@t-online.de

www.zaen.org 